\title{
The influence of stellar wind conditions on the detectability of planetary radio emissions
}

\author{
J.-M. Grießmeier ${ }^{1}$, U. Motschmann ${ }^{1}$, G. Mann ${ }^{2}$, and H. O. Rucker ${ }^{3}$ \\ ${ }^{1}$ Institut für Theoretische Physik, Technische Universität Braunschweig, Mendelssohnstraße 3, 38106 Braunschweig, \\ Germany \\ e-mail: j-m.griessmeier@tu-bs.de;u.motschmann@tu-bs.de \\ 2 Astrophysikalisches Institut Potsdam, An der Sternwarte 16, 14482 Potsdam, Germany \\ e-mail: gmann@aip.de \\ 3 Institut für Weltraumforschung, Österreichische Akademie der Wissenschaften, Schmiedlstrasse 6, 8042 Graz, Austria \\ e-mail: rucker@oeaw.ac.at
}

Received 8 September 2004 / Accepted 21 February 2005

\begin{abstract}
Magnetized giant exoplanets in close orbits around their host star are expected to be strong nonthermal radio emitters. The anticipated radio flux is strong enough to allow its detection on Earth using the next generation of instruments. However, the measured quantity will not be the planetary radio flux but the sum of planetary and stellar emission. We compare the expected stellar and planetary radio signal for stellar systems of different ages. Solar-like stellar wind parameters as well as conditions corresponding to the young solar system (i.e. with increased stellar wind density and velocity) are considered. For young stellar systems, conditions appear to be more favorable than for older stellar systems. It is shown that configurations exist where the separation of the planetary signal from the stellar emission seems feasible.
\end{abstract}

Key words. planetary systems - magnetic fields - radiation mechanisms: non-thermal - Sun: radio radiation radio continuum: stars

\section{Introduction}

When considering "direct detection" of extrasolar planets, one has always to keep in mind that it is at present not possible to resolve an extrasolar planetary system. Thus an observer will always see the combined signal of the central star and of the planet(s). This is true for observations in all spectral ranges, but the intensity ratio of stellar to planetary emission varies. From the calculation of theoretical spectra for wide-separation ( $>0.2 \mathrm{AU}$ ) extrasolar giant planets, Burrows et al. (2004) deduce a flux ratio of $\geq 10^{8}$ in the visible range and $\geq 10^{4}$ for infrared emission. For closer separations, they find a flux ratio of $10^{3}$ for the mid-infrared. The situation is different for the lowfrequency radio range. Planetary radio emission is dominated by powerful nonthermal emission generated by the cyclotronmaser-instability (CMI). The solar radio emission - which will serve as the main example of stellar radio emission throughout this paper - consists of a quiet background (produced by thermal bremsstrahlung) plus a rich spectrum of radio bursts (caused by nonthermal electrons). The difference in generation mechanism allows for a much more favorable intensity ratio in the spectral range considered here, thus making it easier to separate the stellar and the planetary radio emission, as will be explained in detail below.

For most of the discussion we will assume the star to be similar to the sun. In Sect. 2.2 a type of stellar radio emission which does not exist on the sun is presented. For close-in extrasolar giant planets much higher flux densities are expected when compared to the radio planets of the solar system (e.g. Farrell et al. 1999; Zarka et al. 2001). These estimations are reviewed and revised in this work; in addition, we show that the temporal evolution of the stellar wind as presented by Grießmeier et al. (2004) has to be taken into account. We also compare our results to those of the recent study of Lazio et al. (2004).

In Sect. 2 we will treat different kinds of solar (Sect. 2.1) and stellar (Sect. 2.2) radio emission. Section 3 briefly describes the flux density spectrum of Jupiter (Sect. 3.1) and discusses the radio flux expected from different extrasolar planets under present-day stellar wind conditions (Sect. 3.2). Section 4 expands this discussion by taking into account the stellar wind evolution with time. It will be shown how this affects planetary radio emission. In Sect. 5 the stellar and planetary flux densities are compared. Section 6 closes with a few concluding remarks.

\section{Stellar radio flux}

\subsection{Solar radio emission}

The solar radio flux is composed of different components, not all of which are always present. The components differ in intensity and rate of occurrence and are caused by different emission 


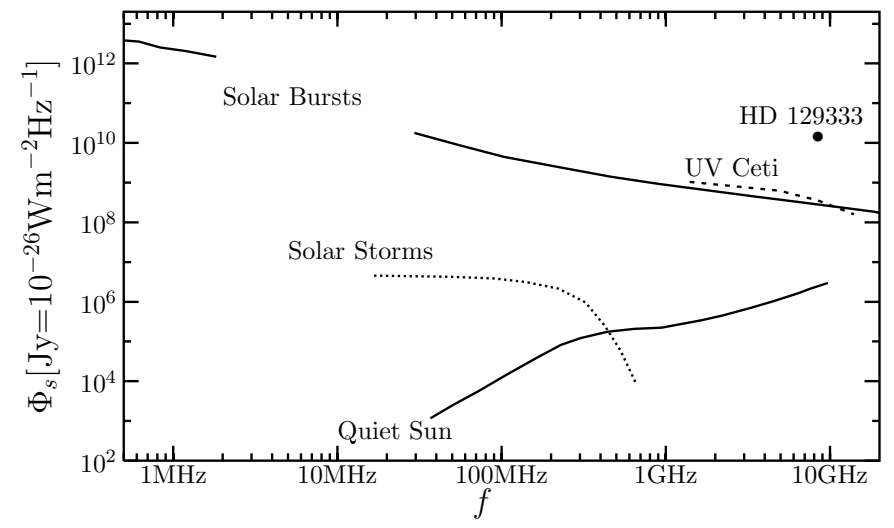

Fig. 1. Solar radio spectrum according to Boischot \& Denisse (1964) (dotted line) and Nelson et al. (1985) (solid lines). The quiescent stellar emission of the dG0e star HD 129333 (=EK Dra) measured at $8.4 \mathrm{GHz}$ (Güdel et al. 1995) as well as that of the dM5.5e star UV Cet (short dashed line, from Güdel \& Benz 1996) are normalized to a distance of $1 \mathrm{AU}$ for comparison (see Sect. 2.2).

mechanisms. Three different emission mechanisms are important (Warmuth \& Mann 2005):

- thermal bremsstrahlung generated by free electrons accelerated by the Coulomb fields of ions;

- gyro emission caused by electrons spiraling around magnetic field lines;

- coherent plasma emission close to the local electron plasma frequency or its harmonic excited by fast electrons.

In the following, the different components contributing to the solar radio flux are discussed in terms of their emission mechanism, commonness, peak intensity and relevance concerning the radio detection of extrasolar planets. Figure 1 shows the intensity of the quiet sun and the observed maximum intensity of solar radio bursts as well as that of noise storms.

The quiet sun emission is caused by thermal bremsstrahlung due to electron-ion collisions in the ionized plasma. Observations at different frequencies typically yield information about different layers in the star. The higher the frequency $f$, the denser (and lower) the generating layer may have been. Most of the observed quiet sun radio emission comes from the solar atmosphere (and not from deep inside the sun).

The slowly varying component (not shown in Fig. 1) is mainly due to gyrosynchrotron emission from regions of hot and dense plasma in the corona e.g. over sunspots (Warmuth \& Mann 2005). This leads to a flux density variation by a factor of $\sim 2$ at centimetre and decimetre wavelengths (Sheridan $\&$ McLean 1985). It has a periodicity of 27 days due to solar rotation (Boischot \& Denisse 1964) and varies over the sunspot cycle. While the quiet sun emission is randomly polarized (Sheridan \& McLean 1985), the emission in the centimetric range is often circularly polarized, which can only be explained by the strong magnetic fields of the sunspots (Boischot \& Denisse 1964).

Solar radio bursts are generated by high-frequency plasma oscillations excited by suprathermal electrons. These plasma oscillations have to be converted into electromagnetic radiation. Solar radio bursts typically have much higher flux densities than the quiet sun emission. They are observed in the whole frequency range, but they are more intense in the low frequency domain (see Fig. 1). The emission takes place close to the electron plasma frequency or its harmonics (Melrose 1985).

Solar radio bursts are usually partially circularly polarized. Some types of solar radio bursts are briefly presented in the following; a more complete review is given by Warmuth \& Mann (2005). Type I bursts only occur in large groups. These Noise Storms are described below. Type II bursts are generated by magnetohydrodynamic shock waves caused by a disturbance moving with super-Alfvénic velocity. Suprathermal electrons in the shock-front region excite Langmuir waves which are converted to electromagnetic radiation. Type II bursts display a detailed fine structure (see e.g. Mel'nik et al. 2004). Polarization is similar to type III bursts (see below). Type III bursts, characteristic of the impulsive phase of solar flares, are the most common flare-associated bursts. They are generated by relativistic electrons (typically $v \approx 0.3 c$, see Warmuth \& Mann 2005). Because of the high particle velocity, a large frequency drift $\mathrm{d} f / \mathrm{d} t$ is a characteristic feature of type IIIBursts. For this type of emission, radio waves are emitted not only at the fundamental frequency of plasma waves, but also at their second harmonic (Bougeret et al. 1984). The polarization degree ranges from weak $(<0.15)$ to moderately high $(\sim 0.5)$. Non-flare related type III bursts are found in type III storms (see below). The broadband emission of a type IV burst is caused by energetic electrons trapped in a closed magnetic structure. Some of these structures are stationary, others move slowly upward, leading to a slow frequency drift. Type V bursts are continuum emissions over a wide frequency range. They follow type III bursts and typically have the opposite circular polarization than the preceding type III burst.

Noise storms are frequently the dominant component of solar radio emission for wavelengths between 1 and $10 \mathrm{~m}$. Both flare and non-flare related noise storms exist. Two types of storms are distinguished, which are named type I storms and type III storms after the type of radio bursts associated with them. Although the radio flux density associated with a type I noise storm is far below that of a radio burst, it can be 1000 times that of the quiet sun. Due to their occurrence rate and their duration, noise storms significantly contribute to the signal detected: near solar maximum, noise storms occur about $10 \%$ of the time (Hjellming 1988). The typical duration of a noise storm is between a few hours and several days (Boischot $\&$ Denisse 1964; Warmuth \& Mann 2005). Type I noise storms consist of a slowly varying broadband continuum plus shortlived bursts. The emission of type I storms is highly circularly polarized (Boischot \& Denisse 1964; Kai et al. 1985). Type III storms are not associated with flares; they are connected to type III bursts (see above). Type III storms can also include continuum emission in addition to bursts, but these continua only have a low intensity (Kai et al. 1985). There often is a temporal relationship between type I storms and type III storms, possibly due to a common exciting agent (Kai et al. 1985). Type III storms always have the same polarization as type I storms, but the degree of polarization is usually much lower for type III storms. 


\subsection{Stellar radio emission}

Some stars are continuously emitting much more energy at radio frequencies than the sun. These radio luminosities can be 2-3 orders of magnitudes higher than the quiet sun (Benz 1993). This kind of emission is probably due to nonthermal processes (possibly gyrosynchrotron emission of energetic electrons); to emphasize the different generation mechanism of this emission with respect to the quiet sun emission, the term quiescent radio emission was introduced. There is no corresponding radiation on the sun. The typical variation of the quiescent emission is about $50 \%$ on a time scale of hours, and it has a low degree of polarization (Benz 1993). Unfortunately, measurements of stellar radio spectra are limited to a few frequencies. Also, no data are available for frequencies below $1 \mathrm{GHz}$. Figure 1 shows a spectrum for the quiescent stellar emission of the dM5.5e star UV Ceti (Güdel \& Benz 1996, dataset 4), normalized to a distance of 1 AU. The stellar distance was taken to be $2.627 \mathrm{pc}$ (calculated from Gliese \& Jahreiß 1991). Güdel \& Zucker (2000) fitted four-point VLA radio spectra to the gyrosynchrotron model of White et al. (1989). This fit indicates that for UV Cet, the maximum of the emission probably lies above $1 \mathrm{GHz}$, so that lower flux densities can be expected for lower frequencies. For frequencies below this maximum, theory predicts that the intensity is proportional to $f^{2.5}$, while in reality exponents between 0 and 10 can be found (Benz 1993).

Also, stars with even higher quiescent radio flux exist. The quiescent radio flux decreases with increasing stellar age (Güdel et al. 1998). For this reason, the emission of a young star can serve as an upper limit for quiescent emission. Figure 1 shows the quiescent stellar emission of the young (approx. 70 Myr, see Dorren \& Guinan 1994) dG0e star HD 129333 (=EK Draconis) as measured at the frequency of $8.4 \mathrm{GHz}$ (Güdel et al. 1995), scaled to a distance of 1 AU. We will use the emission of HD 129333 as the upper limit to the contribution of the quiescent emission.

It is known that stellar flares can be much more energetic than solar flares; stellar flares with $10^{4}$ times the radio flux of the largest solar radio burst have been observed. These flares are often completely circularly polarized (Güdel et al. 1989; Benz 1993). The influence of both stellar flares and quiescent radio emission on the detectability of planets is discussed in Sect. 5.

Some very large flares (up to $10^{7}$ times more energetic than the largest solar flare) on solar-like stars could possibly be caused by the interaction of a normal $\mathrm{G}$ dwarf and a magnetized close-in extrasolar planet (Rubenstein \& Schaefer 2000). However, so far only nine of these transient extreme events have been detected (Schaefer et al. 2000). For this reason, they do not present a systematic problem for the discrimination of stellar and planetary radio emission.

\section{Planetary radio flux}

\subsection{Jupiter's radio emission}

The first measurement of Jupiter's radio emission (the strongest planetary radio emission we know) was made by

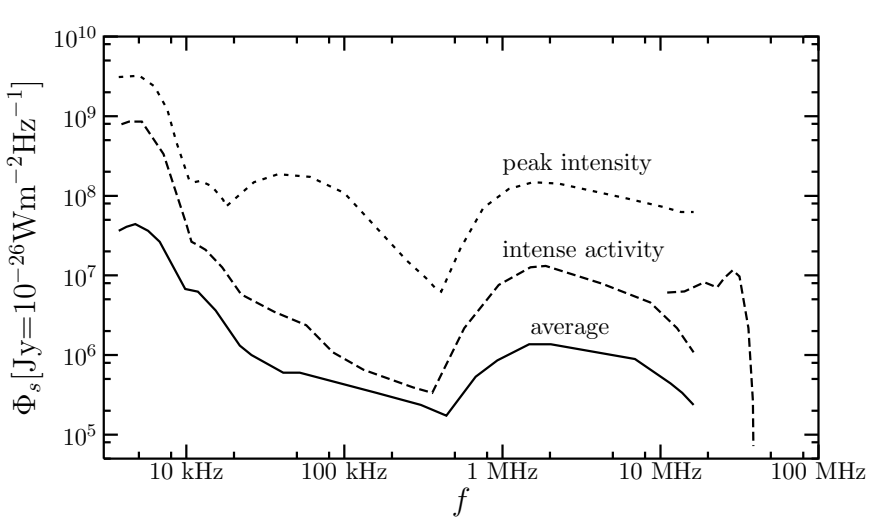

Fig. 2. Jupiter radio spectrum based on Cassini RPWS data (Zarka et al. 2004), normalized to a distance of 1 AU. Solid line: rotation averaged emission. Dashed line: rotation averaged emission at times of intense activity. Dotted line: peak intensities during active periods. The high-frequency data are taken from Zarka et al. (1995) and correspond to times of intense emission (see Zarka et al. 2004).

Burke \& Franklin (1955) at a frequency of $22 \mathrm{MHz}$. Due to the Earth's ionosphere, frequencies below 5-10 MHz (Zarka et al. 1997) are not accessible to ground-based observations. This explains why radio emissions from other planets of the solar system (including the radio emission from the Earth's magnetosphere) were unknown at that time. The full radio spectrum of Jupiter could only be determined years later by the PRA experiment on both Voyager spacecraft (Zarka 1992). About two days of Voyager data (obtained from a distance of 100-500 planetary radii) were used to compute the spectrum, which was first published in 1992. Recently, the spectrum was recalculated with much more accuracy using Cassini-RPWS data (Zarka et al. 2004). Figure 2 is based on that spectrum. Unfortunately, Cassini-RWPS data are only available for $f \leq 16 \mathrm{MHz}$. For higher frequencies, spectral data from Zarka et al. (1995) are shown, corresponding to periods of intense activity (Zarka et al. 2004). It can be seen that the peak flux densities can be up to 100 times the average values. The observed spectrum is highly time-dependent, e.g. through solar wind variability (Gurnett et al. 2002) and also depends on the observer's position (due to beaming effects). To facilitate the comparison with the solar radio emission of Sect. 2, the flux density scale is normalized to $1 \mathrm{AU}$ distance.

The high frequency cutoff in the spectrum shown in Fig. 2 can be explained as follows. Typically, the source region is located between 2 and 4 planetary radii. It has been found that the radio emission is produced close to the local electron gyrofrequency along auroral fieldlines. Thus, the highest frequency emission will be generated at the location with the strongest magnetic field, i.e. closest to the planet's surface. This yields the "high-frequency cutoff" around $40 \mathrm{MHz}$ in Fig. 2. A more complete discussion of the different components of Jupiter's radiation can be found in Zarka (1998), updated in Zarka et al. (2004). Note that planetary radio emission is strongly circularly polarized (Zarka 1992, 1998). 


\subsection{Exoplanetary radio emission}

Already before the discovery of the first extrasolar planet around a star in 1995 (Mayor \& Queloz 1995), attempts were made to discover the radio emission of extrasolar planets. So far, however, these efforts have been unsuccessful (Yantis et al. 1977; Winglee et al. 1986; Zarka et al. 1997; Bastian et al. 2000; Farrell et al. 2003; Lazio et al. 2004). One of the many possible reasons for the current non-detection given by Bastian et al. (2000) is the lack of sensitivity in the appropriate frequency range. This will be verified in this subsection and in Sect. 4.

It is clear that the radio emission of a planet in an extrasolar planetary system can differ considerably from Jupiter's radio emission. A planet in a close-in orbit ( $d \leq 0.1 \mathrm{AU})$ around its central star (a so-called "Hot Jupiter") is subject to strong tidal dissipation, leading to gravitational locking on a very short timescale (Seager \& Hui 2002). For a hypothetical Jupiter-like planet orbiting a solar twin at $0.05 \mathrm{AU}$, the synchronisation timescale is approximately $2 \times 10^{6}$ years. For gravitationally locked planets the rotation period is equal to the orbital period, and fast rotation is not possible. Commonly employed scaling-laws for the planetary magnetic moment (e.g. Cain et al. 1995; Grießmeier et al. 2004) always yield a magnetic moment rapidly decreasing with decreasing rotation rate. This influence of tidal locking on the planetary magnetic moment is examined in detail by Grießmeier et al. (2004).

In Table 1 the planetary parameters required for the magnetic moment estimation as well as the resulting magnetic moments (upper and lower limits) are compared for those planets where the radius is either known (from transit observations) or reasonably well constrained by theoretical models ( $\tau$ Boo, see Burrows et al. 2000). This choice was made because information on the planetary radius allows an improved estimation of the planetrary magnetic moment (Grießmeier et al. 2004). The quantities are given in units normalized to Jupiter (denoted by subscript J), with $R_{\mathrm{J}}=71490 \mathrm{~km}, M_{\mathrm{J}}=$ $1.9 \times 10^{27} \mathrm{~kg}, \omega_{\mathrm{J}}=1.76 \times 10^{-4} \mathrm{~s}^{-1}, \mathcal{M}_{\mathrm{J}}=1.5 \times 10^{27} \mathrm{Am}^{2}$. The planetary radius $R$ and mass $M$ was taken from Cody \& Sasselov (2002) for HD 209458b, from Torres et al. (2004) for OGLE-TR-56b, from Bouchy et al. (2004) for OGLE-TR-113b and from Moutou et al. (2004) for OGLE-TR-132b. For the mass of $\tau$ Boo only the lower limit is known $\left(M \sin i=4.38 M_{\mathrm{J}}\right.$, Leigh et al. 2003), and the radius is presently not accessible to measurement. Theoretical models by Burrows et al. (2000) yield an upper limit for the radius of $1.58 R_{\mathrm{J}}$ for a planetary mass of $7 M_{\mathrm{J}}$ and of $1.48 R_{\mathrm{J}}$ for a mass of $10 M_{\mathrm{J}}$. The most probable radius seems to be $1.2 R_{\mathrm{J}}$ (Leigh et al. 2003). Because of this uncertainty, we calculate the magnetic moment for three different cases: a relatively "light" planet $\left(M=4.4 M_{\mathrm{J}}\right.$ and $\left.R=1.2 R_{\mathrm{J}}\right)$, a "medium" planet $\left(M=7.0 M_{\mathrm{J}}\right.$ and $\left.R=1.58 R_{\mathrm{J}}\right)$, and a "heavy" planet $\left(M=10.0 M_{\mathrm{J}}\right.$ and $\left.R=1.48 R_{\mathrm{J}}\right)$. For each of these three model cases, the upper and lower limit for the magnetic moment $\mathcal{M}$ was calculated. The highest upper limit (i.e. for the "heavy" case) and the lowest lower limit (i.e. for the "light" case) are given in Table 1 . The value of $\omega$ was calculated from the orbital period assuming tidal locking. The core density $\rho_{\mathrm{c}}$ is proportional to the mean density $\bar{\rho}$ of the planet $\left(\rho_{\mathrm{c}} \propto \bar{\rho}=3 M / 4 \pi R^{3}\right)$. See Grießmeier et al. (2004) for additional details.

The magnetic dipole moment values were obtained exactly as in Grießmeier et al. (2004). For the magnetic moment scaling, the size of the dynamo-region $r_{\mathrm{c}}$ is required. Two different scaling laws were suggested for this quantity: one based only on the mass of the planet (Curtis \& Ness 1986) and one based on both planetary mass and radius (Grießmeier et al. 2004). Both approaches lead to unphysical results for the case of $\tau$ Bootes: one finds $r_{\mathrm{c}}>R$. In these cases, we set $r_{\mathrm{c}}=R$ for all subsequent calculations. This approach may seem arbitrary, but it is reasonable to assume a monotonous increase in $r_{\mathrm{c}} / R$ with planetary mass $M$. Thus, the real value for $\tau$ Boo will lie in the range $0.9<r_{\mathrm{c}} / R<1.0$, and the error made by assuming $r_{\mathrm{c}}=R$ is tolerable.

Not only is the size of the magnetic moment and thus of the magnetosphere very different for a "Hot Jupiter". Also, the stellar wind is much denser because of the close proximity of the star. Both these effects will have an influence on the radio power emitted from an extrasolar planet, as will be seen in the following.

A simple way to estimate the total emitted radio power of planets within the solar system was originally suggested by Desch \& Kaiser (1984). This idea was later applied to the expected radio emission from extrasolar planets by Farrell et al. (1999). The total power emitted in the radio range, $P_{\text {rad }}$, is probably roughly proportional to the total power incident on the magnetosphere, $P_{\text {input }}$ (Zarka et al. 2001):

$P_{\text {rad }} \propto P_{\text {input }}$

The energy source is believed to be either the kinetic energy of the solar wind (Desch \& Kaiser 1984; Farrell et al. 1999) or the magnetic energy flux (Zarka et al. 2001; Farrell et al. 2004). In this work, we discuss only the kinetic energy model; applications to the magnetic energy model will be the subject of further studies.

The energy input into the magnetosphere is proportional to the cross-section of the magnetosphere as seen from the star, determined by the magnetospheric radius $R_{\mathrm{M}}$ :

$P_{\text {input }}=\epsilon \frac{m n_{0}}{2 d^{2}} R_{\mathrm{M}}^{2} \pi v^{3}$,

where $m$ is the mass of the stellar wind protons, $v$ is their bulk velocity and $n_{0} / d^{2}$ is their number density at a distance $d$ from the central star, where $d$ is measured in AU. As most of the protons are deflected around the magnetosphere and only a certain portion of the total solar wind kinetic energy is transferred to the magnetosphere an additional proportionality constant $\epsilon$ is introduced. If magnetic connection is considered to be the dominant energy input mechanism, $\epsilon \approx 1 / 5$ for the Earth (Hill 1979). In the following estimation, $\epsilon$ will be considered to be the same for all planets, so that its precise value is not required.

The magnetospheric radius is determined from the pressure balance at the substellar point, i.e.

$m n v^{2} \propto \frac{\boldsymbol{B}_{\mathrm{p}}^{2}}{2 \mu_{0}}$. 
Table 1. Parameters for some "Hot Jupiters". The values used for the calculation of the magnetic moments are given in units normalized to Jupiter. The lower part shows the scaling results for a stellar system of $4.6 \mathrm{Gyr}$ age: planetary dipole moment, total emitted radio power, radio flux at $1 \mathrm{AU}$ from the planet, radio flux at Earth (distance $s$ ) and maximum frequency. Values calculated for Jupiter are given for comparison. Sources of parameters: ${ }^{(a)}$ Burrows et al. (2003), ${ }^{(b)}$ Konacki et al. (2003), ${ }^{(c)}$ Melo et al. (2004), ${ }^{(d)}$ Moutou et al. (2004), ${ }^{(e)}$ Leigh et al. (2003), ${ }^{(f)}$ Cody \& Sasselov (2002), ${ }^{(g)}$ Torres et al. (2004), ${ }^{(h)}$ Bouchy et al. (2004). See text for details.

\begin{tabular}{|c|c|c|c|c|c|c|}
\hline & Jupiter & HD 209458b & OGLE-TR-56b & OGLE-TR-113b & OGLE-TR-132b & $\tau$ Boo \\
\hline$s$ (distance from Earth) [parsec] & - & $47.3^{a}$ & $1500^{b}$ & $370^{c}$ & $2500^{d}$ & $15.6^{e}$ \\
\hline$d$ (semimajor axis) $[\mathrm{AU}]$ & 5.2 & 0.045 & $0.0225^{g}$ & $0.0228^{h}$ & 0.0306 & $0.0489^{e}$ \\
\hline$R$ (radius) $\left[R_{\mathrm{J}}\right]$ & 1 & $1.42^{f}$ & $1.23^{g}$ & $1.08^{h}$ & $1.13^{d}$ & $1.2 \ldots 1.58$ \\
\hline$M$ (mass) $\left[M_{\mathrm{J}}\right]$ & 1 & $0.69^{f}$ & $1.45^{g}$ & $1.35^{h}$ & $1.19^{d}$ & $4.4 \ldots 10.0$ \\
\hline$\omega$ (rotation rate) $\left[\omega_{\mathrm{J}}\right]$ & 1 & 0.12 & 0.34 & 0.29 & 0.24 & 0.12 \\
\hline$\rho_{\mathrm{c}}\left(\right.$ core density) $\left[\rho_{c, \mathrm{~J}}\right]$ & 1 & 0.24 & 0.78 & 1.07 & 0.82 & $1.77 \ldots 3.1$ \\
\hline $\mathcal{M}$ (magnetic moment) $\left[\mathcal{M}_{\mathrm{J}}\right]$ & 1 & $0.005 \ldots 0.10$ & $0.4 \ldots 0.8$ & $0.5 \ldots 0.9$ & $0.2 \ldots 0.6$ & $0.5 \ldots 2.7$ \\
\hline$P_{\text {rad }}$ (emitted radio power) $\left[10^{14} \mathrm{~W}\right]$ & $2.1 \times 10^{-3}$ & $0.037 \ldots 0.26$ & $1.6 \ldots 2.7$ & $1.8 \ldots 2.7$ & $0.74 \ldots 1.3$ & $0.69 \ldots 2.1$ \\
\hline$\Phi_{\mathrm{AU}}$ (flux density at $1 \mathrm{AU}$ ) $\left[10^{10} \mathrm{Jy}\right]$ & $5.1 \times 10^{-3}$ & $17 \ldots 50$ & $14 \ldots 18$ & $9.3 \ldots 11$ & $8.4 \ldots 11$ & $4.0 \ldots 9.7$ \\
\hline$\Phi_{s}($ flux density at distance $s)[\mathrm{mJy}]$ & - & $1.8 \ldots 5.3$ & $O\left(10^{-3}\right)$ & $O\left(10^{-2}\right)$ & $O\left(10^{-3}\right)$ & $3.9 \ldots 9.4$ \\
\hline$f_{\mathrm{c}}^{\max }$ (maximum frequency) $[\mathrm{MHz}]$ & 23 & $0.04 \ldots 0.84$ & $4.9 \ldots 10.4$ & $8.7 \ldots 16$ & $3.6 \ldots 8.9$ & $6.7 \ldots 19$ \\
\hline
\end{tabular}

$\boldsymbol{B}_{\mathrm{p}}(r)$ is the planetary magnetic field and $\mu_{0}$ is the vacuum permeability. Assuming a zonal dipole, the magnetic field strength $B_{\mathrm{p}}$ on the magnetosphere (i.e. at planetocentric distance $R_{S}$ ) depends on the planetary magnetic dipole moment $\mathcal{M}$ as

$B_{\mathrm{p}} \propto \frac{\mathcal{M}}{R_{s}^{3}}$.

Inserting Eq. (4) into Eq. (3) this leads to

$R_{s} \propto \mathcal{M}^{1 / 3}\left(n_{0} v^{2}\right)^{-1 / 6} d^{1 / 3}$

where the stellar wind density $n$ was replaced by $n=n_{0} d^{-2}$. Here $n_{0}$ is the stellar wind density at a given stellar distance (e.g. at $1 \mathrm{AU}$ ). The more detailed calculation in Grießmeier et al. (2004, Sect. 4) includes the effect of magnetospheric currents, but leads to the same result. The only difference is another constant of proportionality.

According to observations and models, $R_{\mathrm{M}} \approx 2 R_{S}$ for Jupiter (Joy et al. 2002). As a first-order approximation, selfsimilarity is satisfied (Vogt \& Glassmeier 2001), so that a fixed ratio can be assumed for all planets: $R_{\mathrm{M}} \propto R_{S}$. Note that in one case (for HD 209458b with $\mathcal{M}=0.005 \mathcal{M}_{\mathrm{J}}$ ), we obtained $R_{\mathrm{M}}<R$. In that case we set $R_{\mathrm{M}}=R$ for all further calculations, because the magnetosphere cannot be compressed to sizes smaller than the planetary radius.

The fact that $R_{\mathrm{M}} \propto R_{S}$ together with Eqs. (5) and (2) can then be inserted into Eq. (1), resulting in

$P_{\text {rad }} \propto \mathcal{M}^{2 / 3} n_{0}^{2 / 3} v^{7 / 3} d^{-4 / 3}$.

A similar result was obtained by Zarka et al. (1997). From Eq. (6) it is clear that the total emitted radio power increases for decreasing orbital distance. This is because the higher kinetic energy flux dominates over the magnetospheric compression (i.e. the smaller cross-section of the magnetosphere). As described above, the size of the magnetic moment depends on the rotation state of the planet. Depending on the type of planet, a higher radio flux can possibly be obtained from fast rotating planets just outside the tidal locking zones of their star.

The total emitted power is now normalized to the radio power emitted by the planet Jupiter, so that Eq. (6) becomes

$P_{\mathrm{rad}}=\left(\frac{\mathcal{M}}{\mathcal{M}_{\mathrm{J}}}\right)^{2 / 3}\left(\frac{n_{0}}{n_{0, \mathrm{~J}}}\right)^{2 / 3}\left(\frac{v}{v_{\mathrm{J}}}\right)^{7 / 3}\left(\frac{d}{d_{\mathrm{J}}}\right)^{-4 / 3} P_{\text {rad, } \mathrm{J}}$

Here, $\mathcal{M}_{\mathrm{J}}=1.5 \times 10^{27} \mathrm{Am}^{2}, n_{0, \mathrm{~J}}=10^{7} \mathrm{~m}^{-3}, v_{\mathrm{J}}=400 \mathrm{~km} \mathrm{~s}^{-1}$, $d_{\mathrm{J}}=5.2 \mathrm{AU}$ are Jupiter's magnetic moment, the solar wind number density and velocity at $1 \mathrm{AU}$ and Jupiter's distance from the Sun, respectively. The total radio flux emitted by Jupiter $P_{\text {rad,J }}$ is calculated from Zarka et al. (2004). As suggested by Zarka et al. (2004), the measured contribution for the DAM contribution was doubled to account for the fact that only part of the DAM-band was measured. Three different values can be defined. The average total power is given by $P_{\text {rad,J }}=3.1 \times 10^{10} \mathrm{~W}$. During periods of high activity, the average total emitted radio power is $P_{\text {rad,J }}=2.1 \times 10^{11} \mathrm{~W}$. The peak power is given by $P_{\mathrm{rad}, \mathrm{J}}=1.1 \times 10^{12} \mathrm{~W}$. In the following, we will use the average radio power during periods of high activity. The radio power calculated for different close-in extrasolar giant planets is given in Table 1. Two values are given; the lower radio power corresponds to the small magnetic moment limit, and the higher value is determined by the upper limit for the magnetic moment from Table 1.

From the total emitted radio power, the radio flux at a given distance can be calculated. Similarly to Farrell et al. (1999) we calculate the radio flux as

$\Phi=\frac{P_{\mathrm{rad}}}{\Omega s^{2} \Delta f}$ 
$\Omega$ is the solid angle of the beam. According to Zarka et al. (2004), one can use $\Omega=1.6$ for the dominating contributions of Jupiter's radio emission. This value will be adopted in the following. It corresponds to a hollow cone of half-angle aperture $\sim 75^{\circ}$ with a cone mantle thickness of $15^{\circ}$. The distance of the given stellar system from Earth is denoted by $s$, and $\Delta f$ is the emission bandwidth. We assume

$$
\Delta f=0.5 f_{\mathrm{c}}^{\max }=\frac{e B_{\mathrm{p}}^{\max }}{4 \pi m_{\mathrm{e}}},
$$

where the maximum cyclotron frequency $f_{\mathrm{c}}^{\max }$ is determined by the maximum magnetic field strength $B_{\mathrm{p}}^{\max }$ close to the polar cloud tops (Farrell et al. 1999). The electron's charge is $e$, and $m_{\mathrm{e}}$ is its mass. A planet with a strong magnetic field is a much more powerful radio emitter than a planet with a weak field, but it emits in a much broader frequency band. Similarly to Eq. (4), the polar magnetic field strength can be expressed by

$$
B_{\mathrm{p}}^{\max }=\frac{\mu_{0}}{4 \pi} \frac{2 \mathcal{M}}{R_{\mathrm{p}}^{3}}
$$

Inserting Eq. (10) into Eq. (8) then yields

$$
\Phi=\frac{8 \pi^{2} m_{\mathrm{e}} R_{\mathrm{p}}^{3} P_{\mathrm{rad}}}{e \mu_{0} \Omega s^{2} \mathcal{M}}
$$

Obviously, a planet with a small magnetic moment, large radius (at fixed magnetic moment) close to a star with a dense and fast stellar wind will show the strongest nonthermal radio emission. With Eq. (7) for $P_{\text {rad }}$ it can be seen that

$\Phi \propto R_{\mathrm{p}}^{3} \mathcal{M}^{-1 / 3} n_{0}^{2 / 3} v^{7 / 3} d^{-4 / 3}$.

Apparently, planets for which a strong magnetic moment is expected are not automatically the best candidates for the search for radio emission. On the other hand, for a weakly magnetized planet, $f_{\mathrm{c}}^{\max }$ will be small, making ground-based detection impossible (ionospheric cutoff). Table 1 gives $\Phi_{s}$, the radio flux expected at Earth, as well as $\Phi_{\mathrm{AU}}$, the radio flux normalized to $1 \mathrm{AU}$. The latter value is a measure of the strength of planetary radio emission (which has to be compared to the expected stellar emission), while the former determines whether a given planet can be detected by radio telescopes. Because it is important to have frequencies above the ionospheric cutoff limit $(5-10 \mathrm{MHz})$ for ground-based detection, Table 1 also gives the maximum emission frequencies. Note that the upper limit for the flux and maximum frequency are given by the lower limit for the magnetic moment and vice versa. For $\tau$ Bootes, the limits given in Table 1 are found by comparing the limits obtained for the three different models.

If we compare our results for $\tau$ Bootes with the result of Farrell et al. (1999, Table 1), we can see that in the present work the total radio power is smaller by a factor of $\sim 4$, while the flux is higher by a factor of $\sim 4$. The values for the radio power differ because of the different magnetic moments, while the flux densities are different because we use a different magnetic moment, a smaller value of $\Omega$ and a smaller value for $\Delta f$. Thus, for average conditions, the results are approximately comparable. While in Farrell et al. (1999) variations of $\Omega$ and of stellar wind velocity are suggested to potentially increase the total radio flux by up to three orders of magnitude, we believe that the peak power will not be more than one order of magnitude above the value given in Table 1 (e.g. if $P_{\text {rad, J }}=1.1 \times 10^{12} \mathrm{~W}$ is used rather than $\left.P_{\text {rad,J }}=2.1 \times 10^{11} \mathrm{~W}\right)$. For this reason, the peak radio power (and the peak radio flux) differ considerably. Note that so far, stellar wind conditions corresponding to a stellar system age of $4.6 \mathrm{Gyr}$ were compared. The correct age will be taken into account in Sect. 4.

In comparison to the recent study of Lazio et al. (2004) we note that Table I of that work gives the peak power, while we use the average power during periods of high intensity. Lazio et al. (2004) take the peak power to be two order of magnitude above average, leading to a peak emission power much larger than that of the current study. As far as the maximum emission frequency is concerned, we note that the frequency given by Lazio et al. (2004) is higher by a factor of 3 . This is due to the fact that they use the magnetic moment scaling of Blackett (1947), which was later experimentally disproved (Blackett 1952). Due to the larger exponent in $r$, Blackett's laws leads to an overestimation of the planetary magnetic moment for large planets. In addition, Lazio et al. (2004) make use of the mass-radius relation $R \propto M^{1 / 3}$. Especially for planets with large masses like $\tau$ Boo, this produces unrealistically large planetary radii, magnetic moments, radio fluxes and emission frequencies. Note that a good estimation of the emission frequency is particularly important because a difference of a small factor can make the difference between radiosignals above and below the ionospheric cutoff.

The radio flux $\Phi_{s}$ from Table 1 has to be compared to the detection limit of various radio-telescopes: the UTR-2 detector in Kharkov presently has a sensitivity of $25 \mathrm{Jy}$ (e.g. Rucker 2002). This detector will be improved in the near future using modern digital techniques, where a new backend facility called Robin 2 (Rucker 2002) will improve the sensitivity down to $100 \mathrm{mJy}$. The extremely ambitious project of a LOw Frequency ARray (LOFAR) will provide a sensitivity of $1 \mathrm{mJy}$ (Kassim et al. 2004).

It can be seen that the radio flux from the planet HD 209458 b could in principle be detectable by LOFAR, but that the frequency is below the ionospheric cutoff. The reason for this is the weak magnetic field due to synchronous rotation (tidal locking). The planets OGLE-TR-56b, OGLE-TR-113b and OGLE-TR-132b are very far away. Even if the planets are relatively powerful radio emitters and ionospheric cutoff is not a problem, the radio flux at Earth is much too low to allow detection. Although these planets are not good candidates for radio observations, they can serve as representatives of a certain class of planets. Note that non-transiting but otherwise similar planets could exist much closer to the solar system. For such a planet, radio detection would be possible. Finally, it can be seen that the radio emission expected for $\tau$ Boo lies both above the ionospheric cutoff frequency and above the detection limit for LOFAR, so that radio emission from that planet may be detectable in the near future. 
Table 2. Scaling output for a planet like $\tau$ Bootes at different stellar system ages (4.6 Gyr, 1.0 Gyr and 0.7 Gyr). See text for details.

\begin{tabular}{lccc}
\hline \hline$\tau$ Bootes & $4.6 \mathrm{Gyr}$ & $1.0 \mathrm{Gyr}$ & $0.7 \mathrm{Gyr}$ \\
\hline$v(t)$ (stellar wind velocity) $\left[\mathrm{km} \mathrm{s}^{-1}\right]$ & 400 & 731 & 840 \\
$n_{0}(t)$ (stellar wind density at $\left.1 \mathrm{AU}\right)\left[\mathrm{m}^{-3}\right]$ & $1.0 \times 10^{7}$ & $9.5 \times 10^{7}$ & $15.9 \times 10^{7}$ \\
\hline $\mathcal{M}$ (magnetic moment) $\left[\mathcal{M}_{\mathrm{J}}\right]$ & $0.5 \ldots 2.7$ & $0.5 \ldots 2.7$ & $0.5 \ldots 2.7$ \\
$P_{\text {rad }}$ (emitted radio power) $[\mathrm{W}]$ & $0.69 \times 10^{14} \ldots 2.1 \times 10^{14}$ & $1.3 \times 10^{15} \ldots 3.8 \times 10^{15}$ & $2.5 \times 10^{15} \ldots 7.3 \times 10^{15}$ \\
$\Phi_{\mathrm{AU}}$ (flux density at $\left.1 \mathrm{AU}\right)[\mathrm{Jy}]$ & $4.0 \times 10^{10} \ldots 9.7 \times 10^{10}$ & $7.4 \times 10^{11} \ldots 1.8 \times 10^{12}$ & $1.4 \times 10^{12} \ldots 3.5 \times 10^{12}$ \\
$\Phi_{s}$ (flux density at distance $\left.s\right)[\mathrm{Jy}]$ & $0.0039 \ldots 0.0094$ & $0.071 \ldots 0.17$ & $0.14 \ldots 0.33$ \\
$f_{\mathrm{c}}^{\max }$ (maximum frequency) $[\mathrm{MHz}]$ & $6.7 \ldots 19$ & $6.7 \ldots 19$ & $6.7 \ldots 19$ \\
\hline
\end{tabular}

\section{Radio emission and stellar system age}

Recently, stellar mass loss rates of main sequence $\mathrm{G}$ and $\mathrm{K}$ stars were estimated by using recent indirect stellar wind observations (Wood et al. 2002). This allowed the calculation of the stellar wind evolution (Grießmeier et al. 2004, Sect. 3.2); this calculation was later limited to stellar systems with an age $\geq 0.7$ Gyr (Lammer et al. 2004). It could be shown that the stellar wind velocity of a young star (1 Gyr after reaching the main sequence) is about twice as high as the velocity of today's solar wind (at 4.6 Gyr). Likewise, the density of the stellar wind of a young $\mathrm{G}$ or $\mathrm{K}$ star is about one order of magnitude higher. This has two effects: first, it compresses the magnetosphere, as can be seen from Eq. (5). Secondly, the increased stellar wind parameters also increase the energy input into the magnetosphere, see Eq. (2). It can be seen from Eq. (7) that a denser and faster stellar wind will lead to stronger planetary radio emission. This effect is important because the age of extrasolar planet host stars vary. For the star HD 209458, an age of between 4 and 7 Gyr is assumed, with 5.2 Gyr as a likely value (Mazeh et al. 2000; Cody \& Sasselov 2002). For OGLE-TR-56 the age is given as $3 \pm 1$ Gyr (Sasselov 2003). $\tau$ Bootes has an age of approximately $1 \pm 0.6$ Gyr (Fuhrmann et al. 1998). Using Eqs. (16) and (17) from Grießmeier et al. (2004) for $v(t)$ and $n(t)$, respectively, and Eqs. (7) and (11) from above, the total emitted radio power and the radio flux density are calculated and compared in Table 2 for stellar system ages of 4.6 Gyr, 1.0 Gyr and 0.7 Gyr. To facilitate the comparison, we also give $v$ and $n$ for the different cases. The planetary radius, magnetic moment and semimajor axis are assumed to be constant, so that the time evolution is caused by the variation of $v$ and $n$.

The lower limit for the radio flux expected from a planet like $\tau$ Bootes $\mathrm{b}$ at different stellar system ages as well as the radio flux of Jupiter are compared in Fig. 3. Note that if an age of $4.6 \mathrm{Gyr}$ is assumed for $\tau$ Bootes, the expected radio flux is underestimated by over one order of magnitude. Concerning the current non-detection of radio emission from $\tau$ Bootes, we suggest that the main problem is the relatively low maximum frequency of $19 \mathrm{MHz}$ of the emission as compared to the measurements at $f \geq 74 \mathrm{MHz}$ (Winglee et al. 1986; Bastian et al. 2000; Farrell et al. 2003; Lazio et al. 2004). The observations of Zarka et al. (1997) are in a more promising frequency range

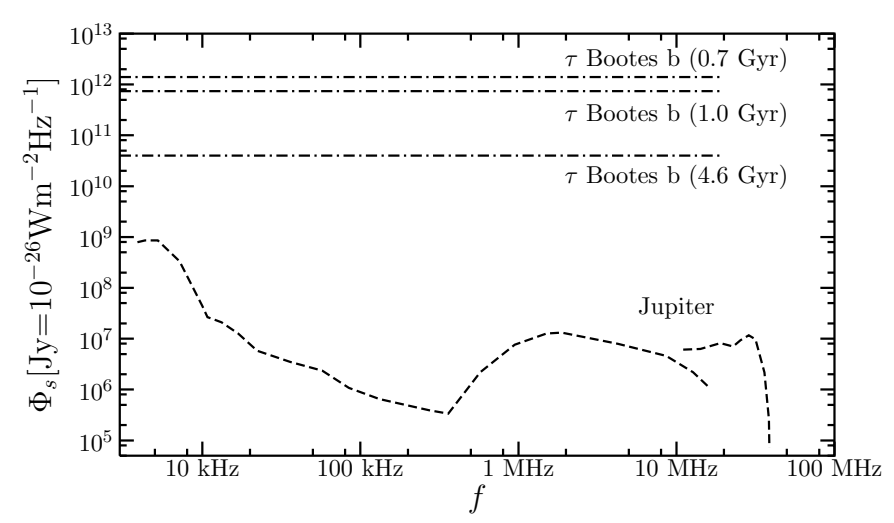

Fig. 3. Comparison of the radio flux measured from Jupiter (cf. Fig. 2) according to Zarka et al. $(1995,2004)$ at periods of intense activity (dashed lines) and the lower limit for the radio flux emission from a planet like $\tau$ Bootes $\mathrm{b}$ at different ages (dash-dotted lines). All values are normalized to a distance of $1 \mathrm{AU}$.

(between 7 and $35 \mathrm{MHz}$ ), but the sensitivity of the UTR-2 detector was not sufficient. Similarly, the measurements by Yantis et al. (1977) were not sensitive enough.

\section{Radio comparison}

In this section we compare the flux density of different radio sources. Again, to facilitate the comparison, all flux densities in Fig. 4 are normalized to a distance of 1 AU. Note that this is done because for an extrasolar planetary system the star and the planet will have the same distance to a detector at Earth.

We first consider the contribution of the galactic background. It is known that the galactic background depends on the viewing direction. It can be measured by a second measurement of the sky close to the extrasolar system and then be subtracted from the signal received from the system. For example, the UTR-2 radio telescope in Kharkov can be used in a two-beam mode with one of the beams directly on the radio source and the second beam pointing $1^{\circ}$ away from the first beam (Zarka et al. 1997). Another option would be to remove the sky background by interferometry, see e.g. Nelson et al. (1985).

If Jupiter's radio emissions is compared to the sun's emission, one clearly notes that the planetary emission is far more powerful than the quiet sun emission. Thus the question is not 


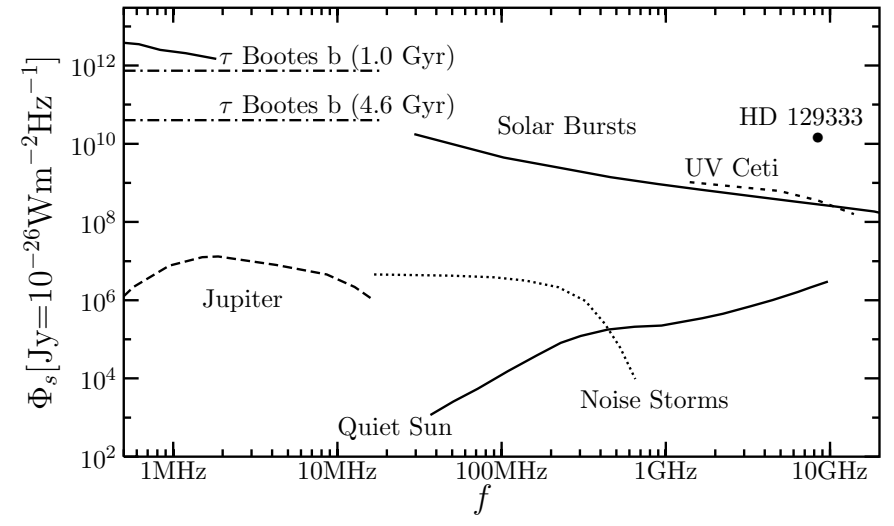

Fig. 4. Solar radio data according to Boischot \& Denisse (1964) (dotted line) and Nelson et al. (1985) (solid lines). Jupiter radio flux during periods of intense activity (cf. Fig. 2) according to Zarka et al. (2004) (dashed line). Also shown: quiescent stellar emission from UV Ceti (short-dashed line) and HD 129333 (see Sect. 2.2) and lower limit for the radio flux expected from $\tau$ Bootes (dash-dotted lines, see Sects. 3.2 and 4). All values are normalized to a distance of $1 \mathrm{AU}$.

whether an extrasolar planet would be detectable against a sunlike star (during quiet conditions), but rather whether such a quiet star would be detectable against the radiation of such a planet. The slowly varying component (not shown in Fig. 4) does not contribute much to the solar flux in the spectral range where planetary emission is expected.

The quiescent emission some stars exhibit could in principle be problematic. Although no measurements are available for low frequencies $(\sim 1 \mathrm{GHz})$, it seems likely that the flux levels are much lower at frequencies relevant for planetary detection ( $\leq 25 \mathrm{MHz}$ ). Also, quiescent radio emission seems to be connected to stellar X-ray emission (Güdel et al. 1995). Thus, the comparison of the radio emission and the X-ray emission might serve as an indicator of whether the source of the radio emission is likely to be the star or not. Also, the low degree of polarization of the quiescent emission (see Sect. 2.2) as opposed to the planetary radio emission will prove to be an important diagnostic tool.

Due to their relatively low occurrence-rate (about $10 \%$ of the time near solar maximum, see Sect. 2.1), noise storms are not very important for the case where a Jupiter-like planet is to be detected around a Sun-like star. They could have an influence in systems where either the planetary radio emission is weaker than Jupiter's, or in cases of a star showing more activity than the sun. In these cases statistical considerations would be required, and the quiet star level would have to be evaluated carefully. On the other hand, for close-in extrasolar planets, emission much stronger than Jupiter's is expected. Thus the contribution of noise storms probably is negligible.

Stellar radio bursts are another matter. In the solar system, they are far more powerful than any planetary radio emission. The question arises as to whether the latter could be separated from such a bursty background. Fortunately, these radio bursts do not occur all the time. Although type IV emission can last for several days, it only occurs with a rate of approximately 3 per month during sunspot maximum. Type III emission happens much more frequently (up to 1400 per month at sunspot maximum), but its duration is limited to a few seconds (Boischot \& Denisse 1964). Thus it can be hoped that using statistical arguments the stellar bursts can be separated from planetary emission. If one admits the possibility that the beaming direction of extrasolar planetary radio emission could be very different from what we see in the solar system, it could be worth while to look at secondary eclipses of transiting planets as suggested by Richardson et al. (2003). In this case different spectra may be obtained during secondary eclipse ("star only"spectra when the planet passes behind the star) and off-eclipse ("star plus planet"-spectra). The noisy background of the stellar radio bursts could then be reduced by statistics, i.e. by observing not one but many eclipses of an appropriate planet. Of course, a star with little activity in the radio spectrum (like the Sun near solar minimum) is always preferable.

The planetary radio emission of some extrasolar planets may be much stronger than Jupiter's emission. For example, for a system similar to $\tau$ Bootes, the radio emission will be several orders of magnitude stronger than Jupiter's emission (see Fig. 3). It can be seen that detection is more likely for young stellar systems, where the stellar wind is denser and faster than for today's Sun. The radio emission from $\tau$ Bootes at its present age $(1.0 \mathrm{Gyr})$ is much stronger than the contributions of the galactic background, the quiet sun emission, solar noise storms, and also the assumed maximum quiescent radio emission. Some stellar radio bursts will still be more intense than the planetary emission; depending on the occurrence rate of radio bursts on the star, this might be more or less problematic. This will be especially true if the planets host star exhibits very strong stellar flares.

Even in cases where the combined stellar/planetary radio signal contains major contributions from the planet, one also requires some means to separate the two contributions. There are several ways that this can be achieved. Firstly, it is known that planetary radio emission is highly polarized (Zarka 1998). This is not the case for the quiet sun radio emission or the quiescent radio emission (see Sect. 2). Secondly, the bursty component could possibly be discriminated by occultation during secondary eclipses. Thirdly, for planets not subjected to tidal locking (i.e. far enough from their star) rotation rates could help to distinguish the two components. For the solar system it is known that the radio emissions are modulated with the planetary rotation, which is of the order of hours, whereas the stellar rotation is measured in days. This method will fail in the few cases where the stellar and the planetary rotation are synchronized. As discussed by Pätzold \& Rauer (2002), some measurements of the stellar rotation period of $\tau$ Bootes seem to indicate that this is the case (this is also suggested by Leigh et al. 2003), while other values suggest that the star is rotating more slowly. But even if the method is not applicable to $\tau$ Bootes, the modulation with the rotation period may be very useful for other stellar systems.

\section{Conclusion}

We have compared the radio flux expected from close-in giant extrasolar planets with the different radiation components of their host stars. For this purpose, a planetary radio scaling 
calibrated by the radio spectrum of Jupiter obtained from recent Cassini data (Zarka et al. 2004) was presented. Care must be taken not to produce unphysical results: The condition $r_{\mathrm{c}} \leq$ $R \leq R_{\mathrm{M}}$ always should be checked. Several extrasolar planets were discussed; it was shown that due to tidal locking, many of the close-in planets $(d \leq 0.1 \mathrm{AU})$ are only weakly magnetized, leading to a maximum frequency below the ionospheric cutoff limit. Other planets have a stronger magnetic moment (either because of their even smaller orbital radius, like OGLETR-113 b, or due to their larger mass, like $\tau$ Bootes). For these planets, detection is possible in principle, provided the planet is not located too far away from the Earth (which is the case for OGLE-TR-113 b). On the other hand, similar planets may well exist closer to Earth. It was also shown that because of the stellar wind evolution, the radio flux from young systems is much more important. Thus, the best candidates for planetary radio detection seem to be young, massive "Hot Jupiters" not too far away from the Earth. It was shown that at least for one system ( $\tau$ Bootes), intense radio emission is expected, which should in principle be detectable on Earth with the next generation of radio telescopes (LOFAR), and maybe even before that. Past observations like those of Zarka et al. (1997) and Bastian et al. (2000) were either not sensitive enough or were limited to higher frequencies. Note that a space-based radio observatory would avoid the restriction of the frequency range caused by the ionospheric cutoff.

Different methods were presented which might prove useful for the discrimination of the different contributions of star and planet to the total radio signal. It was shown that for the $\tau$ Bootes system, the contribution of the planetary radio signal will dominate over the stellar radio flux, except for strong radio bursts. If the star's radio bursts are comparable to solar radio bursts, it should be possible to extract the planetary radio characteristics from the combined stellar and planetary signal.

This comparison shows that radio observations of an extrasolar planetary systems will yield information not only on the stellar emission, but also on the planetary radio emission in the near future.

Acknowledgements. We would like to thank the referee for comments and suggestions. J.-M.G. thanks A. Benz for additional comments. This research has made use of the VizieR catalogue access tool, CDS, Strasbourg, France.

\section{References}

Bastian, T. S., Dulk, G. A., \& Leblanc, Y. 2000, ApJ, 545, 1058

Benz, A. 1993, Plasma Astrophysics: Kinetic Processes in Solar and Stellar Coronae (Dordrecht: Kluwer Academic Publishers) Blackett, P. M. S. 1947, Nature, 159, 658

Blackett, P. M. S. 1952, Phil. Trans. R. Soc. A, 245, 309

Boischot, A., \& Denisse, J. F. 1964, Adv. in Electronics and Electron. Phys., 20, 147

Bouchy, F., Pont, F., Santos, N. C., et al. 2004, A\&A, 421, L13

Bougeret, J.-L., King, J. H., \& Schwenn, R. 1984, Sol. Phys., 90, 401

Burke, B. F., \& Franklin, K. L. 1955, J. Geophys. Res., 60, 213

Burrows, A., Guillot, T., Hubbard, W. B., et al. 2000, ApJ, 534, L97
Burrows, A., Sudarsky, D., \& Hubbard, W. B. 2003, ApJ, 594, 545

Burrows, A., Sudarsky, D., \& Hubeny, I. 2004, ApJ, 609, 407

Cain, J. C., Beaumont, P., Holter, W., Wang, Z., \& Nevanlinna, H. 1995, J. Geophys. Res., 100, 9439

Cody, A. M., \& Sasselov, D. D. 2002, ApJ, 569, 451

Curtis, S. A., \& Ness, N. F. 1986, J. Geophys. Res., 91, 11003

Desch, M. D., \& Kaiser, M. L. 1984, Nature, 310, 755

Dorren, J. D., \& Guinan, E. F. 1994, ApJ, 428, 805

Farrell, W. M., Desch, M. D., \& Zarka, P. 1999, J. Geophys. Res., 104, 14025

Farrell, W. M., Lazio, T. J., Desch, M. D., Bastian, T., \& Zarka, P. 2003, in Scientific Frontiers in Research of Extrasolar Planets, ed. D. Deming, \& S. Seager, ASP Conf. Ser., 294

Farrell, W. M., Lazio, T. J. W., Zarka, P., et al. 2004, Planet. Space Sci., 52, 1469

Fuhrmann, K., Pfeiffer, M. J., \& Bernkopf, J. 1998, A\&A, 336, 942

Gliese, W., \& Jahreiß, H. 1991, Preliminary Version of the Third Catalogue of Nearby Stars, Tech. rep., On: The Astronomical Data Center CD-ROM: Selected Astronomical Catalogs, Vol. I; ed. L. E. Brotzmann, S. E. Gesser, NASA/Astronomical Data Center, Goddard Space Flight Center, Greenbelt, MD

Grießmeier, J.-M., Stadelmann, A., Penz, T., et al. 2004, A\&A, 425, 753

Güdel, M., \& Benz, A. O. 1996, in Radio Emission from the Stars and the Sun, ed. A. R. Taylor, \& J. M. Paredes, ASP Conf. Ser., 93, 303

Güdel, M., Benz, A. O., Bastian, T. S., et al. 1989, A\&A, 220, L5

Güdel, M., Guinan, E. F., \& Skinner, S. L. 1998, in Cool Stars, Stellar Systems and the Sun, ed. R. A. Donahue, \& J. A. Bookbinder, ASP Conf. Ser., 154, 1041

Güdel, M., Schmitt, J. H. M. M., \& Benz, A. O. 1995, A\&A, 302, 775

Güdel, M., \& Zucker, A. 2000, in Highly Energetic Physical Processes and Mechanisms for Emission from Astrophysical Plasmas, ed. P. C. H. Martens, \& S. Tsuruta, IAU Symp., 195, 393

Gurnett, D. A., Kurth, W. S., Hospodarsky, G. B., et al. 2002, Nature, 415,985

Hill, T. W. 1979, in Proc. of Magnetospheric Boundary Layers Conference, Alpbach, ESA SP-148, 325,

Hjellming, R. M. 1988, in Galactic and Extragalactic Radio Astronomy, 2nd ed., ed. G. L. Verschuur, \& K. I. Kellermann, Astronomy and Astrophysics Library (Berlin: Springer-Verlag), 381

Joy, S. P., Kivelson, M. G., Walker, R. J., et al. 2002, J. Geophys. Res., SMP 17, 107

Kai, K., Melrose, D. B., \& Suzuki, S. 1985, in Solar Radiophysics, ed. D. J. McLean, \& N. R. Labrum (Cambridge: Cambridge University Press), 415

Kassim, N. E., Lazio, T. J. W., Ray, P. S., et al. 2004, Planet. Space Sci., 52, 1343

Konacki, M., Torres, G., Jha, S., \& Sasselov, D. D. 2003, Nature, 421, 507

Lammer, H., Ribas, I., Grießmeier, J.-M., et al. 2004, Hvar Obs. Bull., 28,139

Lazio, T. J. W., Farrell, W. M., Dietrick, J., et al. 2004, ApJ, 612, 511

Leigh, C., Cameron, A. C., Horne, K., Penny, A., \& James, D. 2003 , Mon. Not. R. Astron. Soc., 344, 1271

Mayor, M., \& Queloz, D. 1995, Nature, 378, 355

Mazeh, T., Naef, D., Torres, G., et al. 2000, ApJ, 532, L55

Mel'nik, V. N., Konovalenko, A. A., Rucker, H. O., et al. 2004, Sol. Phys., 222, 151

Melo, C., Bouchy, F., Pont, F., et al. 2004, The ESO Messenger, 116, 32

Melrose, D. B. 1985, in Solar Radiophysics, ed. D. J. McLean, \& N. R. Labrum (Cambridge: Cambridge University Press), 177 
Moutou, C., Pont, F., Bouchy, F., \& Mayor, M. 2004, A\&A, 424, L31

Nelson, G. J., Sheridan, K. V., \& Suzuki, S. 1985, in Solar Radiophysics, ed. D. J. McLean, \& N. R. Labrum (Cambridge: Cambridge University Press), 113

Pätzold, M., \& Rauer, H. 2002, ApJ, 568, L117

Richardson, L. J., Deming, D., Wiedemann, G., et al. 2003, ApJ, 584, 1053

Rubenstein, E. P., \& Schaefer, B. E. 2000, ApJ, 529, 1031

Rucker, H. O. 2002, ESA, SP-518, 421

Sasselov, D. D. 2003, ApJ, 596, 1327

Schaefer, B. E., King, J. R., \& Deliyannis, C. P. 2000, ApJ, 529, 1026

Seager, S., \& Hui, L. 2002, ApJ, 574, 1004

Sheridan, K. V., \& McLean, D. J. 1985, in Solar Radiophysics, ed. D. J. McLean, \& N. R. Labrum (Cambridge: Cambridge University Press), 443

Torres, G., Konacki, M., Sasselov, D. D., \& Jha, S. 2004, ApJ, 609, 1071

Vogt, J., \& Glassmeier, K.-H. 2001, Adv. Space Res., 28, 863
Warmuth, A., \& Mann, G. 2005, in Lecture Notes in Physics, Space Weather: The physics behind the slogan (Springer), 656, 49

White, S. M., Kundu, M. R., \& Jackson, P. D. 1989, ApJ, 225, 112

Winglee, R. M., Dulk, G. A., \& Bastian, T. S. 1986, ApJ, 209, L59

Wood, B. E., Müller, H.-R., Zank, G. P., \& Linsky, J. L. 2002, ApJ, 574, 412

Yantis, W. F., III, W. I. S., \& Erickson, W. C. 1977, BAAS, 9, 453

Zarka, P. 1992, Adv. Space Res., 12, (8)99

Zarka, P. 1998, J. Geophys. Res., 103, 20159

Zarka, P., Pedersen, B. M., Lecacheux, A., et al. 1995, in Neptune and Triton, ed. D. P. Cruikshank (Tuscon: Univ. of Ariz. Press), 341

Zarka, P., Queinnec, J., Ryabov, B. P., et al. 1997, in Planetary Radio Emissions IV, ed. H. O. Rucker, S. J. Bauer, \& A. Lecacheux (Vienna: Austrian Academy of Sciences Press), 101

Zarka, P., Treumann, R. A., Ryabov, B. P., \& Ryabov, V. B. 2001, Ap\&SS, 277, 293

Zarka, P., Cecconi, B., \& Kurth, W. S. 2004, J. Geophys. Res., 109, A09S15 